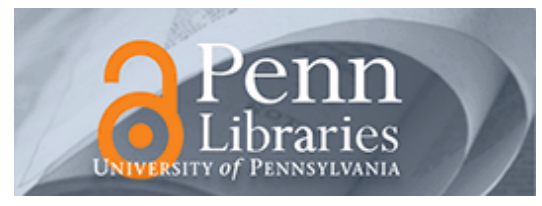

University of Pennsylvania

ScholarlyCommons

June 2002

\title{
Thin Absorbing Screens Using Metamaterial Surfaces
}

\author{
Nader Engheta \\ University of Pennsylvania, engheta@seas.upenn.edu
}

Follow this and additional works at: https://repository.upenn.edu/ese_papers

\section{Recommended Citation}

Nader Engheta, "Thin Absorbing Screens Using Metamaterial Surfaces", . June 2002.

Copyright 2002 IEEE. Reprinted from the 2002 IEEE Antennas and Propagation Society International Symposium Volume 2, pages 392-395.

Publisher URL: http://ieeexplore.ieee.org/xpl/tocresult.jsp?isNumber=21869\&page=6

This material is posted here with permission of the IEEE. Such permission of the IEEE does not in any way imply IEEE endorsement of any of the University of Pennsylvania's products or services. Internal or personal use of this material is permitted. However, permission to reprint/republish this material for advertising or promotional purposes or for creating new collective works for resale or redistribution must be obtained from the IEEE by writing to pubs-permissions@ieee.org. By choosing to view this document, you agree to all provisions of the copyright laws protecting it.

This paper is posted at ScholarlyCommons. https://repository.upenn.edu/ese_papers/13

For more information, please contact repository@pobox.upenn.edu. 


\title{
Thin Absorbing Screens Using Metamaterial Surfaces
}

\author{
Abstract \\ Metamaterial surfaces can be conceptualized by 2-dimensional periodic arrangement of many small flat \\ inclusions on an otherwise homogeneous host surface. Electromagnetic properties of such metamaterial \\ plates, which can indeed be regarded as frequency-selective surfaces, are influenced by the shape and \\ geometry of these inclusions. When a metamaterial surface is closely placed above a perfectly \\ conducting plate, at certain band of frequency, this structure may possess high surface impedance at its \\ top surface, and thus providing a high-impedance ground plane (HIGP). The center frequency and \\ bandwidth over which such high-impedance electromagnetic surface is achieved depend on inclusion \\ shapes and compositions, among other parameters. By placing a resistive sheet on top of this surface, \\ we can achieve a thin structure that can be an efficient absorber for incident electromagnetic energy. \\ Comments \\ Copyright 2002 IEEE. Reprinted from the 2002 IEEE Antennas and Propagation Society International \\ Symposium Volume 2, pages 392-395. \\ Publisher URL: http://ieeexplore.iee.. rg/xpl/tocresult.jsp?isNumber=21869\&page=6 \\ This material is posted here with permission of the IEEE. Such permission of the IEEE does not in any way \\ imply IEEE endorsement of any of the University of Pennsylvania's products or services. Internal or \\ personal use of this material is permitted. However, permission to reprint/republish this material for \\ advertising or promotional purposes or for creating new collective works for resale or redistribution must \\ be obtained from the IEEE by writing to pubs-permissions@ieee.org. By choosing to view this document, \\ you agree to all provisions of the copyright laws protecting it.
}




\title{
Thin Absorbing Screens Using Metamaterial Surfaces
}

\author{
Nader Engheta \\ University of Pennsylvania \\ Department of Electrical Engineering \\ Philadelphia, Pennsylvania 19104, U.S.A. \\ Tel: +1-215-898-9777, Fax: +1-215-573-2068 \\ E-mail: sngghelägec.upenn.edu, URL: http://wwh.ee.upenn.edu/ engheta/
}

\section{Abstract}

Metamaterial surfaces can be conceptualized by 2-dimensional periodic arrangement of many small flat inclusions on an otherwise homogeneous host surface. Electromagnetic properties of such metamaterial plates, which can indeed be regarded as frequencyselective surfaces, are influenced by the shape and geometry of these inclusions. When a metamaterial surface is closely placed above a perfectly conducting plate, at certain band of frequency, this structure may possess high surface impedance at its top surface, and thus providing a high-impedance ground plane (HIGP). The center frequency and bandwidth over which such high-impedance electromagnetic surface is achieved depend on inclusion shapes and compositions, among other parameters. By placing a resistive sheet on top of this surface, we can achieve a thin structure that can be an efficient absorber for incident electromagnetic energy.

\section{Introduction}

Study of electromagnetic properties of complex media and complex surfaces has recejved considerable attention in the past several decades (see, e.g., [1]-[8]). Researchers in several groups all over the world have been investigating various features of electromagnetic composite media, also known as metamaterials, which can possess novel electromagnetic properties, not readily available in nature, but physically realizable. Owing to their exciting features, these "artificial" materials can offer interesting potential applications in antennas, devices and components. Here, we describe one of our ideas, namely the possibility of having thin absorbing screens for electromagnetic energy, as a potential application of metamaterial surfaces.

\section{Thin Absorbing Screens}

As is well known, a highly conductive flat surface has a very low surface impedance, $Z_{x}=0$, that would result in a reflectivity of $R \simeq-1$ for incident wave on the conducting surface. However, if a surface is designed to have a very high surface impedance, the reflectivity for the incident wave on it would be $R=+1$, which effectively suggests that such a surface may act as a "magnetic wall" in contrast to the conventional electric wall for which $R=-1$. These surfaces can obviously have interesting applications. For example, for a surface with $R=+1$, it has been shown that a small dipole antennas can be laid horizontally near the surface, and the image current will be in phase with the antenna current, tesulting in good radiation efficiency (see e.g. [9]). 
Metamaterial surfaces, when they are placed near, and parallel with, a conducting plate may offer an interesting possibility for achieving high-impedance surfaces. For example, let us take a metamaterial surface that can conceptually be made by having many small flat inclusions distributed, in a periodic fashion, on a flat surface. Such a surface can indeed behave as a frequency-selective surface when an incident electromagnetic wave interacts with it. Such a thin metamaterial plate by itself can be characterized electromagnetically by a shunt impedance matrix $\underline{Z}_{\text {shunt }}$. This implies that when an incident plane wave is illuminating this metamaterial plate by itself, the reflected and transmitted waves can be obtained by treating the plate as the shunt impedance $\underline{Z}_{\text {shum }}$. Such shunt impedance does in general depend on frequency and angle of incidence of incoming wave, and the specific form of this function depends on several parameters including the shape, density, and size of the inclusions. Now we place this metamateria] surface in front of a highly conductive metallic plate at a distance $d$, which is assumed to be much smaller than the operating wavelength $\lambda$, with a dielectric layer sandwiched between the metamaterial surface and the metallic plate. (Fig. 1a). The surface impedance at the top surface of this combined layer of metamaterial surface, dielectric spacer, and the ground plane can be obtained using the transmission-line theory as given below:

$$
Z_{\text {surface }}=\frac{j Z_{\text {shum }} \eta_{t d} \tan \left(k_{\text {nd }} d\right)}{Z_{\text {shunt }}+j \eta_{t d} \tan \left(k_{n d} d\right)}
$$

where $Z_{\text {surface }}$ represents the surface impedance at the top surface of the combined layer, $\eta_{s d}$ is the transverse intrinsic impedance of the dielectric layer, and $k_{n d}$ is the nomal component of wave vector in the dielectric layer. (Here for the sake of mathematical simplicity, we assume the metamaterial plate is "isotropic", i.e., $\underline{\underline{Z}}_{\text {shunt }}$ can be written in a scalar form $Z_{\text {shunt }}$. The general anisotropic surface can also be easily treated using the matrix manipulation of transmission-line theory.) As can be seen from the above equation, if the metamaterial surface is designed such that for a given frequency range its equivalent shunt impedance $Z_{\text {shunt }}$ satisfies the following relation

$$
Z_{\text {shunt }}=-j \eta_{t d} \tan \left(k_{n d} d\right)=-j \eta_{t d} k_{n d} d
$$

then the surface impedance $Z_{\text {sufface }}(\omega)$ can in principle attain a very high value in this frequency range. Therefore, the top surface of the combined layer would have a reflectivity of $R=+1$, effectively acting as a magnetic wall. Variation of the equivalent shunt impedance $Z_{\text {shunt }}$ as a function of frequency relies on various parameters among which one should mention the shape of the inclusions. For instance, in our work on wire media, we have obtained interesting variation for $Z_{\text {shunt }}$ for a plane made of many long, parallel, thin wire inclusions [6], which may allow the possibility of achieving the $R=+1[10]$. 


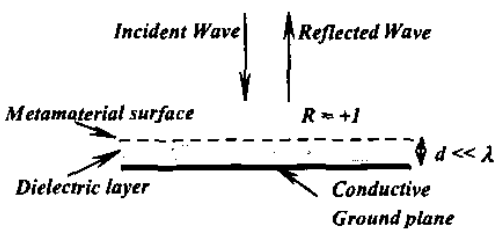

(a)

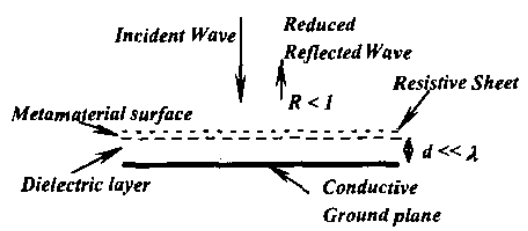

(b)

Fig. 1. Sketch of an idea for application of metamaterial surfaces in thin absorbing screens. Panel (a) shows a metamaterial surface over a thin, grounded dielectric layer. If the metamaterial surface can be designed such that the top surface of this structure has high surface impedance, then the reflection coefficient for the incoming wave will be $R=+1$, resulting in a high value for the total tangential component of electric field on the top surface. As a result, if a resistive sheet with a proper value of resistivity is placed on top of this structure, (Panel (b)), the reflected wave can be reduced appreciably.

For such a combined layer, the reflectivity for the incident wave will then become $R \approx+1$. Now we assume a normally incident plane wave impinging on this structure. (Here, only for the sake of simplicity of the argument, we assume the normally incident wave. We can easily extend this argument to the oblique incidence.) Since $R \cong t 1$, the total tangential component of the electric field and of the magnetic field on the top surface of this structure are, respectively, $E_{t}^{\text {total }} \approx 2 E_{t}^{\text {incident }}$ and $H_{t}^{\text {total }}=0$, so as we said earlier the surface may effectively act as a "magnetic wall". Thus unlike the case of a simple metallic ground plane (i.e., electric wall), here the tangential component of the total electric field is significant on the top surface. If we can now lay a thin layer of resistive sheet on top of this surface (Fig. $\mathrm{lb}$ ), since the electric field is high on this surface, part of the incident energy can be dissipated on this resistive sheet, and thus the reflection coefficient can be reduced appreciably. This can be quantitatively shown by using the transmission-line theory. If the shunt resistance of the resistive sheet is taken to be $r_{\text {resitive }}$, and if we assume that the metamaterial surface can be designed so that the reflectivity for the combined structure (before we put the resistive sheet on) is $R=+1$, then the normal-incidence reflection coefficient after the resistive sheet is a placed on this structure can be obtained as $R=\left(r_{\text {resistive }}-\eta_{o}\right) /\left(r_{\text {resistiviv }}+\eta_{o}\right)$. With the proper choice of the value of resistive sheet impedance, one may then have the possibility of reducing the reflection coefficient and thus reducing the scattering cross section.

This potential idea resembles the idea of single-screen Salisbury shields [11]. However, in the case of Salisbury shields the electric resistive sheet has to be placed at the $\lambda / 4$ distance away from the metallic plate in order to be at the location with the maximum electric field. But the distance $\lambda / 4$ makes the combined structure thick, and thus makes it unsuitable for many applications. However, in our idea described above if the metamaterial surface can be designed to achieve the high surface impedance at the top surface of the structure, then the resistive sheet can be laid right on top of the structure, providing the potential for thin, lightweight absorbing screens. 
In this talk, we will present some of the results of our theoretical analysis on thin absorbing screens, with the metamaterial surfaces being taken to be a wire-medium surface, which can be synthesized by having many long, parallel, thin wire inclusions placed on a flat surface. Other possibilities for metamaterial surfaces with different inclusion geometries will also be mentioned, and mathematical steps, physical insights and intuitive remarks will be given.

\section{References}

[1] J. Brown, "Artificial dielectrics," in Progress in Dielectrics, vol. 2, J. B. Birks and J. H. Schulman, Eds., London: Heywood, 1960, pp. 193-225.

[2] M. M. I. Saadoun and N. Engheta, "A Reciprocal Phase Shifter Using Novel Pseudochiral or $\Omega$ medium," Microwave and Optical Technology Letters, vol. 5, no. 4, pp. 184-188, 1992.

[3] I. V. Lindell, A. H. Sihvola, S. A. Tretyakov, and A. J. Viitanen, Electromagnetic Waves in Chiral and Bi-Isotropic Media, Boston, MA: Artech House, 1994.

[4] C. A. Moses and N. Engheta, "An idea for electromagnetic "feedforwardfeedbackward' media," IEEE Transactions on Antennas and Propagation, vol. 47, no. 5, pp. 918-928, 1999.

[5] D. L. Jaggard, A. R. Mickelson, and C. H. Papas, "On electromagnetic waves in chiral media," Journal of Applied Physics, vol. 18, pp. 21 I-21 6, 1979.

[6] C. A. Moses and N. Engheta, "Electromagnetic Wave Propagation in the Wire Medium: A Complex Medium with Long Thin Inclusions," in the Special Issue of Wave Motion on the topic of "Electrodynamics in Complex Environments", Vol. 34, No. 3, pp. 301-318, September 2001

[7] I. P. Theron and J. H. Cloete, "The optical activity of an artificial non-magnetic uniaxial chiral crystal at microwave frequencies," Journal of Electromagnetic Waves and Applications, vol, 10, pp. 539-561, 1996.

[8] F. Auzanneau and R. W. Ziolkowski, "Theoretical study of synthetic bianisotropic materials, "Journal of Electromagnetic Waves and Applications, vol. 12, p. 353, 1998.

[9] D. Sievenpiper, L. Zhang, R. F. Jimenez Broas, N. G. Alexopolous, and E. Yablonovitch, "High-Impedance Electromagnetic Surface with a Forbidden Frequency Band," IEEE Transactions on Microwave Theory and Techniques, Vol. 47, No. 11, pp. 2059-2074, November 1999.

[10] N. Engheta, "Dipole Radiation near a Wire-Medium Sheet above a Ground Plane" an URSI-B abstract for presentation in the URSI-B session, at the 200I IEEE AP-S International Symposium and USNC/URSI National Radio Science Meeting, in Boston, MA, July 8-13, 2001, page 387 of URSI Digest.

[11] R. L. Fante and M. T. McCormack, "Reflection Properties of the Salisbury Screen," IEEE Transactions on Antennas and Propagation, Vol. Ap-36, pp. 1443$1454,1988$. 